ISSN 2447-9071

doi https://doi.org/10.36414/rbmc.v6i15.38

Contato para correspondência:

Felipe Oppenheimer Torres

E-mail:

felipeotorres2@hotmail.com

Conflito de interesse: Não

Financiamento: Recursos próprios

Recebido: 02/05/2020

Aprovado: 16/06/2020

\section{A utilização de espargidores de solução lacrimogênea durante a resistência passiva}

\section{The use of riot control chemical agents hand-held devices during passive resistance}

Felipe Oppenheimer Torres ${ }^{1}$

${ }^{1}$ Polícia Militar de Santa Catarina - PMSC

\section{Resumo}

O presente artigo tem por objetivo sugerir a padronização, por parte das polícias militares, no atendimento de ocorrências onde o indivíduo abordado esboce resistência passiva. No presente trabalho será sugerida a utilização, em determinadas situações de resistência passiva, do espargidor de solução lacrimogênea para preservar a integridade física do indivíduo abordado, bem como dos agentes de segurança pública envolvidos na ocorrência. Ao longo do artigo serão elencados alguns parâmetros que indicarão a possibilidade de utilização do espargidor de solução lacrimogênea no caso do indivíduo oferecer resistência passiva, bem como serão abordadas características importantes a respeito dos espargidores e a forma correta dos mesmos serem utilizados em uma ocorrência policial.

Palavras-Chave: Espargidor de solução lacrimogênea, Resistência passiva, Uso diferenciado da força.

\begin{abstract}
The present article aims to suggest the standardization, by the military police, in the attendance of occurrences where the individual approached out lines passive resistance. In the present work, it will be suggested the use, in certain situations of passive resistance, of riot control chemical agents hand-held devices to preserve the physical integrity of the approached individual, as well as the public security agents involved in the occurrence. Throughout the article, some parameters will be listed that will indicate the possibility of using the riot control chemical agents handheld devices in case the individual offers passive resistance, as well as important characteristics regarding the hand-held devices and the correct way they will be used in a police occurrence.
\end{abstract}

Keywords: Riot control chemical agents hand-held devices, Passive resistance, Force continuum.

\section{Introdução}

Infelizmente manchetes como"policial é morto com a própria arma durante abordagem em São Paulo"1 estão se tornando cada vez mais frequentes em nossa sociedade. Contudo, ocorrências desse tipo nem sempre são noticiadas, fazendo com que fatalidades como essas passem despercebidas, não dando, dessa forma, a real importância que o caso merece, e, consequentemente, não gerando uma estatística nacional precisa para nos atentarmos a esta vulnerabilidade.

Durante o atendimento de ocorrências o policial muitas vezes se depara com um dilema que pode acarretar a perda do maior bem que temos, a vida. Não é difícil policiais se depararem com ocorrências onde tiveram que pensar e ponderar, por diversas vezes durante o atendimento daquele empenho, se deveria ou não utilizar determinado nível de força para cessar uma ameaça, com receio de poder responder no futuro pelo crime de abuso de autoridade ou algum outro crime mais gravoso. Entretanto, nem sempre o policial terá 
tempo hábil suficiente e proteção física necessária para fazer este juízo de valor, o que pode acarretar na morte do agente de segurança pública por excesso de zelo.

É certo que durante os cursos de formação os policiais são submetidos a um intenso treinamento e a situações que se assemelhem às ocorrências reais que poderão se deparar no dia a dia. Entretanto, por melhor que seja o treinamento, jamais ele será idêntico à ocorrência real, uma vez que existem diversos fatores que influenciarão o desenrolar da ocorrência.

Além das leis, as quais todos os cidadãos devem se submeter, os policiais precisam ainda estar vinculados a princípios, doutrinas e procedimentos que versam exclusivamente sobre a atuação policial, uma vez que existem situações que são muito pontuais e específicas, não cabendo ao legislador federal legislar sobre estes fatos. Baseado nisso, as polícias militares vêm adotando, cada vez com mais frequência, manuais, procedimentos operacionais padrão, diretrizes e portarias que têm o condão de padronizar e direcionar a atuação policial de acordo com o tipo e nível de ocorrência que será atendida, primando sempre pela segurança do cidadão abordado e do policial envolvido na ocorrência.

Nesse contexto, destacamos a doutrina de uso diferenciado da força, doutrina esta que certamente é a base fundamental no atendimento de qualquer ocorrência policial ordinária. Ao longo desse artigo serão definidos alguns conceitos pertinentes a essa doutrina. O principal objetivo desse trabalho é sugerir uma mudança importante no atendimento de ocorrências onde o cidadão abordado ofereça resistência passiva, sugerindo a possibilidade de utilização, por parte do agente de segurança pública, de espargidores de solução lacrimogênea, sendo ortoclorobenzilmalononitrilo (CS) ou oleoresina de capsicum (OC), desde que estejam presentes alguns requisitos que serão abordados ao longo do presente. Essa mudança tem o intuito de resguardar a integridade física do(s) cidadão(s) abordado(s), bem como do(s) policial(ais) envolvido(s) na ocorrência.

\section{Uso diferenciado da força}

Uso diferenciado da força é um conceito que serve para determinar, regular e disciplinar o dever legal do uso da força, atribuído ao Estado por meio da força policial. Consiste num processo de avaliação prévia do policial em relação ao indivíduo suspeito ou infrator, passando pela seleção adequada de opções de força pelo policial, em resposta ao nível de submissão daquele indivíduo, findando na resposta do policial ${ }^{2}$. Ou seja, o nível de força aplicado pelo policial em determinada ocorrência será baseado exclusivamente na resposta oferecida pelo indivíduo abordado.
No mundo existem diversos modelos de uso diferenciado da força: FLECT, PHOENIX, REMSBERG, NASHVILLE, entre outros. No Brasil, o modelo mais utilizado é o FLETC adaptado, que foi desenvolvido em 1992, nos Estados Unidos, pelo Instituto de Treinamento Policial da Universidade de Illinois. Esse modelo é baseado em uma pirâmide dividida em cinco níveis, a qual envolve a percepção do policial quanto à resposta do indivíduo abordado: submissão à ordem, resistência passiva, resistência ativa, agressão física não letal e agressão física-letal. Para cada atitude do cidadão, existe uma ação por parte do policial na mesma ordem: verbalização, controle de contato, controle físico, força não-letal e força letal ${ }^{3}$.

Atualmente, em uma ocorrência policial em que o cidadão abordado apresenta resistência passiva não é padronizada a utilização de espargidores de solução lacrimogênea. Nesse cenário, o policial deve utilizar o nível de força denominado de controle de contato, que consiste em aplicar técnicas de imobilização ou pontos de pressão em determinadas região do corpo que irão ocasionar a consequente submissão do indivíduo abordado.

Ocorre que para ser adotado o nível de força supracitado - controle de contato - o policial obrigatoriamente precisa se aproximar do cidadão para tentar imobilizá-lo. Essa aproximação aumenta em demasiado o risco para o policial, uma vez que nesse cenário o policial necessitará diminuir a distância de segurança para o cidadão que está sendo abordado, sem saber se o mesmo tem conhecimento de alguma arte marcial, ou ainda, se o indivíduo está portando algum tipo de objeto que possa ferir ou até mesmo causar a morte do policial.

Associado ao fato exposto acima, o policial, ao se aproximar do cidadão para imobilizá-lo, obrigatoriamente deixa a sua arma exposta e vulnerável, mesmo que ela esteja no coldre, abrindo a possibilidade do indivíduo apanhar esta arma e utilizar contra o próprio policial, fato este que já ocorreu em diversos estados brasileiros.

Diante dessa possibilidade e realidade, infelizmente, nada distante, a utilização do espargidor de agente químico lacrimogêneo poderá fazer a diferença entre a vida e a morte do policial. Entretanto, não será em qualquer ocorrência em que o indivíduo abordado oferecer resistência passiva que o policial poderá utilizar o espargidor para resguardar a sua segurança.

É de suma importância que o policial tenha conhecimento técnico prévio e possa fazer uma análise da ocorrência apresentada para decidir se é ou não um caso de utilização do espargidor. Antes do policial se aproximar do indivíduo que está oferecendo resistência passiva, o policial deverá fazer um dimensionamento da cena e, baseado nas características da ocorrência, decidir se a mesma pode ser resolvida somente 
com o nível de força de controle de contato ou se deverá utilizar o espargidor de agente químico lacrimogêneo anteriormente ao controle de contato, sempre tendo por norte preservar a integridade física do cidadão e do policial.

Alguns fatores, necessariamente, precisarão ser observados para a utilização do espargidor em uma ocorrência na qual o indivíduo abordado ofereça resistência passiva, como exemplo podemos citar os seguintes:

- Superioridade numérica dos indivíduos que estão oferecendo resistência passiva em relação aos policiais que estão realizando a abordagem;

- Indícios de que o indivíduo que está oferecendo resistência passiva é praticante de alguma arte marcial;

- Desvantagem do policial em relação ao porte físico do indivíduo que está oferecendo resistência passiva;

- Indícios de que o indivíduo que está oferecendo resistência passiva está sob efeito de álcool ou alguma substância entorpecente;

- Indícios de que a aproximação do policial para imobilizar o indivíduo que está oferecendo resistência passiva exponha ambos a algum risco em relação à integridade física;

- Histórico de que o indivíduo que está oferecendo resistência passiva já tenha oferecido resistência em abordagens anteriores.

Importante frisar que os fatores elencados acima são meramente exemplificativos, e não taxativos. Caberá ao policial que está empenhado na ocorrência estudar o cenário para decidir se o caso concreto se enquadra na utilização ou não do espargidor de solução lacrimogênea, sob pena de responder administrativamente e/ou penalmente caso a utilização do espargidor tenha se dado de forma abusiva ou cometido algum excesso.

Deve-se salientar ainda que o policial, na hipótese do cidadão abordado oferecer resistência passiva, antes de utilizar o espargidor, deverá adotar uma verbalização mais enérgica e contundente a fim de tentar convencer o indivíduo a cooperar. Caso a verbalização não surta efeito e o indivíduo não se torne cooperativo, o policial deverá informar ao indivíduo, previamente, a intenção de utilizar o espargidor contendo a solução lacrimogênea, desde que este aviso não coloque em risco a integridade física do policial.

Mais uma vez devemos frisar que a utilização do espargidor de solução lacrimogênea tem o intuito de preservar a integridade física do indivíduo abordado, bem como do policial, se enquadrando assim dentro das normas de Direitos Humanos. A utilização prévia do espargidor, em determinadas ocorrências, permitirá que o policial eventualmente tenha uma vantagem em relação ao indivíduo abordado, diminuindo a possibilidade de ter que entrar em luta corporal com ele, luta essa que pode causar lesões graves no indivíduo e/ou policial, e em um pior cenário, expor a arma de fogo do policial para o indivíduo que está oferecendo resistência.

Uma vez utilizado o espargidor de solução lacrimogênea, o policial tem a obrigação de assim que o indivíduo tiver sido imobilizado e a situação estiver sob controle, promover, na primeira oportunidade que tiver, a descontaminação do indivíduo que foi exposto ao agente químico lacrimogêneo.

Outro ponto de fundamental importância é a forma que o indivíduo exposto ao espargidor de solução lacrimogênea será conduzido, uma vez que dependendo da forma em que ele for posicionado dentro da viatura poderá ocorrer asfixia posicional, ou seja, a posição do corpo irá interferir no fluxo de oxigênio podendo gerar asfixia no indivíduo. Assim, o indivíduo deverá ser colocado na viatura de forma que fique sentado.

Ao utilizar o espargidor de solução lacrimogênea, o policial deverá ter em mente que pretende causar algum dos seguintes efeitos no indivíduo exposto: distrair, desorientar, interromper atividades, dispersar e/ou reduzir a capacidade combativa ou capacidade de reação do sujeito.

\section{Espargidor de agente químico lacrimogêneo e suas características}

Podemos definir agente químico lacrimogêneo como toda substância química produzida com a intenção de debilitar temporariamente um ou mais indivíduos por meio da irritação da pele, olhos e/ou membranas mucosas, onde os efeitos fisiológicos se iniciam imediatamente após a exposição à substância química e os efeitos desaparecem pouco tempo depois de concluída a exposição ao agente químico.

Os espargidores de agente químico lacrimogêneo comercializados no Brasil podem conter o agente químico ortoclorobenzilmalononitrilo (CS) ou oleoresina de capsicum (OC). Em relação ao tamanho, podem ser divididos em espargidores coletivos ou espargidores individuais, de acordo com o peso e a quantidade de solução lacrimogênea que é armazenada no seu interior. Os espargidores comercializados no Brasil ainda apresentam três formas de espargimento: aerossol, espuma ou gel. Os espargidores de espuma ou gel, que causam predominantemente contaminação tópica, são recomendados em ocorrências em que existe a necessidade de se contaminar um indivíduo, sem, contudo, contaminar o ambiente e/ou terceiros que estejam no local. É uma contaminação pontual e seletiva. Já o espargidor de aerossol, que causa contaminação tópica e interna, é recomendável em ocorrências em que não 
existe a preocupação de contaminação de terceiros e/ou do ambiente. O espargidor de aerossol ainda pode apresentar três modelos de espargimento: stream, cone e névoa.

O modelo stream contém apenas líquido e não possui nenhum gás propelente. As partículas são muito grandes ( $>500$ $\mu \mathrm{m})$. Dos três modelos supracitados, o stream é o que possui o maior alcance e é o que sofre menor influência do vento. Entretanto, ele é o que necessita de uma pontaria mais precisa, já que para fazer efeito ele necessariamente precisar atingir os olhos do indivíduo, assim como ocorre nos espargidores de espuma e gel ${ }^{4}$.

$\mathrm{Na}$ outra extremidade, existe o modelo névoa, que é o modelo que possui as menores partículas de aerossol (0,5-50 $\mu \mathrm{m}) 5$. Este modelo necessita de um propelente para ajudar a transportar as partículas no ar, já que as mesmas são muito pequenas, e por isso não conseguem se locomover de forma eficiente no ambiente ${ }^{4}$.

Abaixo, é apresentada um quadro contendo as principais diferenças existentes entre os modelos supracitados:

Quadro 1. Comparativo das características dos modelos de aerossol.

\begin{tabular}{|c|c|c|c|c|c|}
\hline $\begin{array}{l}\text { Modelo } \\
\text { de } \\
\text { Aerossol }\end{array}$ & Alcance & $\begin{array}{c}\text { Precisão no } \\
\text { Espargi- } \\
\text { mento }\end{array}$ & $\begin{array}{l}\text { Contami- } \\
\text { nação de } \\
\text { terceiros }\end{array}$ & Alvo & 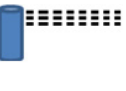 \\
\hline Stream & $\begin{array}{c}\text { Maior } \\
\text { alcance }\end{array}$ & Necessária & Inexistente & $\begin{array}{l}\text { Indivi- } \\
\text { dual }\end{array}$ & \\
\hline Cone & $\begin{array}{l}\text { Interme- } \\
\text { diário }\end{array}$ & $\begin{array}{c}\text { Pouco } \\
\text { necessária }\end{array}$ & Moderada & $\begin{array}{l}\text { Indivi- } \\
\text { dual ou } \\
\text { pequeno } \\
\text { grupo }\end{array}$ & \\
\hline Névoa & $\begin{array}{c}\text { Curto } \\
\text { alcance }\end{array}$ & $\begin{array}{c}\text { Não é } \\
\text { necessária }\end{array}$ & Alta & Multidão & \\
\hline
\end{tabular}

Fonte: Adaptado ${ }^{4}$.

Apesar do modelo aerossol cone ser muito parecido com o modelo aerossol névoa, existe diferença entre os tamanhos das partículas de aerossol. No modelo névoa são bem menores, atingindo o tamanho de $0,5-50 \mu \mathrm{m}^{5}$. Este fato faz com que possa ocorrer um acúmulo maior de agente químico nos pulmões, aumentando, assim, a preocupação com efeitos nocivos no trato respiratório. Destaca-se ainda que os modelos cone e névoa sofrem maior influência por parte de fatores climáticos, como o vento, por exemplo, diferente do modelo stream, que como os espargidores de gel e espuma, são mais resistentes às variações ocasionadas pela natureza.

Contudo, os modelos stream, espuma ou gel devem ser utilizados com uma precaução adicional, pois se no momento em que forem espargidos o indivíduo estiver com a boca aberta, o agente químico poderá ser aspirado, o que poderá acarretar danos aos pulmões. Uma estimativa razoável de limite para ocasionar dano aos pulmões provocado por aspiração de líquido é de $0,1 \mathrm{ml} / \mathrm{kg}$, correspondendo, por exemplo, a $7 \mathrm{ml}$ em um adulto que pesa $70 \mathrm{~kg}^{4}$.

Fator importante na utilização do espargidor é o tamanho das partículas do aerossol, uma vez que dependendo do tamanho das mesmas irão afetar com maior agressividade o trato respiratório do que os olhos, isso porque partículas muito pequenas têm um acúmulo mínimo nos olhos e são transportadas primeiramente para o trato respiratório. O potencial e o grau de irritação no trato respiratório está associado ao tamanho das partículas de aerossol e a concentração de capsaicinóides existentes nas partículas ${ }^{4}$.

Os espargidores individuais devem ser utilizados respeitando a distância mínima de 1 metro do recipiente do espargidor para o indivíduo que se pretende atingir. Já para os espargidores coletivos esta distância é dobrada, devendo ser respeitado o mínimo de 2 metros do recipiente do espargidor para o indivíduo que se pretende atingir. Contudo, caso essa distância não consiga ser respeitada, é recomendável que o policial utilize o espargidor na região superior do tórax do indivíduo, caso o espargidor seja no modelo de aerossol, com o intuito de que o indivíduo se afaste, para que em uma eventual posterior espargida se tenha a distância necessária para atingir o seu rosto. Essas distâncias são importantes, pois garantem que a velocidade do jato do espargidor será menor que $17 \mathrm{~m} / \mathrm{s}$, e velocidades superiores a esta podem gerar danos oculares dependendo da densidade da partícula de aerossol, conforme previsto na tabela abaixo:

Tabela 1. Velocidade das partículas ( $\mathrm{m} / \mathrm{s}$ ) correspondente aos parâmetros de pressão.

\begin{tabular}{|c|c|c|}
\hline Densidade $\mathbf{( k g / \mathbf { m } ^ { \mathbf { 3 } } )}$ & SE $\mathbf{1}$ (20 $\mathbf{~ p s i )}$ & SE 3 (38 psi) \\
\hline 800 & $19 \mathrm{~m} / \mathrm{s}$ & $26 \mathrm{~m} / \mathrm{s}$ \\
\hline 900 & $18 \mathrm{~m} / \mathrm{s}$ & $24 \mathrm{~m} / \mathrm{s}$ \\
\hline 1000 & $17 \mathrm{~m} / \mathrm{s}$ & $23 \mathrm{~m} / \mathrm{s}$ \\
\hline
\end{tabular}

Fonte: Adaptado ${ }^{4}$

SE 1 - lesão controlada que irá se curar sozinha;

SE 3 - efeito potencialmente nocivo à saúde, gerando risco de morte ou risco de lesão permanente. Necessário hospitalização e/ou cuidados especiais.

É importante ainda salientar que os nossos olhos têm um limite na capacidade de armazenamento de fluídos extras. Volumes de fluídos superiores a $30 \mu$ não serão retidos pelo olho e 
irão escorrer para fora dele4. Dessa forma, é recomendável que o espargidor seja acionado utilizando jatos de 0,5 a 1 segundo, evitando, assim, desperdício de material e uma exposição ao agente químico superior à necessária.

Ademais, estudos indicam que quando 1.000 partículas são espargidas do espargidor, aproximadamente 400 partículas de tamanho entre 1-10 $\mu \mathrm{m}$ saiam do recipiente. O número de partículas nesse tamanho permanece inalterado pelo período de até 20 minutos. Isso ocorre porque as partículas maiores encolhem devido à evaporação do solvente contido na partícula, atingindo, assim, o diâmetro supracitado, que consequentemente irão substituir as partículas menores que foram removidas pela ventilação. Dessa forma, conclui-se que uma única espargida de 1 segundo de duração pode gerar efeitos fisiológicos que atingem o pulmão 4 .

Contudo, o policial deverá avaliar o caso concreto para julgar se apenas um acionamento do espargidor na forma explanada acima será suficiente para proporcionar uma maior segurança no momento da imobilização do indivíduo que está oferecendo resistência passiva.

As partículas de aerossol dos modelos stream e cone são emitidas na mesma velocidade, no entanto a pressão ocasionada no olho pelo modelo cone é menor, isso ocorre devido às diferenças nos tamanhos das partículas - $880 \mu \mathrm{m}$ de diâmetro para o modelo cone e $1990 \mu \mathrm{m}$ para o modelo stream. Essa diferença de tamanho faz com que as partículas do modelo cone sofram mais interferência por parte da resistência do $\mathrm{ar}^{4}$.

Tabela 2. Diferença de pressão (PSI) no olho do modelo stream e cone de acordo com variação da distância.

\begin{tabular}{|c|c|c|c|c|c|}
\hline \multirow{2}{*}{ Modelo } & \multicolumn{5}{|c|}{ Definição } \\
\cline { 2 - 6 } & 1 & 2 & 3 & 4 & 5 \\
\hline Stream & 12.05 & 8.76 & 6.08 & 4.23 & 2.34 \\
\hline Cone & 10.8 & 6.5 & 4.2 & 3.8 & 1.8 \\
\hline
\end{tabular}

Fonte: Adaptado ${ }^{4}$

Os espargidores funcionam mediante recipientes pressurizados. A pressão no recipiente é determinada pelo tipo e quantidade de propelente existente. Contudo, essa pressão pode ser influenciada pelas temperaturas do ambiente em que o espargidor é armazenado, uma vez que à medida que a temperatura do recipiente é elevada, a pressão contida no interior do mesmo também sofre um aumento, influenciando, assim, na velocidade das partículas de aerossol caso seja utilizado no momento em que a pressão estiver mais alta por conta do aumento da temperatura. Assim, os espargidores devem ser acondicionados em locais onde não haja um grande aumento de temperatura.

Os espargidores se diferenciam em relação aos órgãos humanos alvo de acordo com o tamanho das partículas formadas. Espargidores que geram partículas com diâmetros maiores do que $100 \mu \mathrm{m}$ têm como alvo primário os olhos. Já partículas menores do que $100 \mu \mathrm{m}$ geralmente não atingem de forma eficaz a superfície do olho, pois estas partículas menores não têm energia cinética suficiente para tal, ao contrário, elas tendem a permanecer no ambiente e circundar o indivíduo ${ }^{4}$.

Por outro lado, partículas com o diâmetro de $100 \mu \mathrm{m}$ são difíceis para serem inaladas, e se forem inaladas, serão filtradas pelo nariz, não atingindo o trato respiratório. As partículas para atingirem o trato respiratório devem possuir diâmetros maiores que $40 \mu \mathrm{m}$. Assim, espargidores que espargem partículas de tamanho menores têm como alvo primário o trato respiratório ${ }^{4}$.

Abaixo, é apresentada um quadro com os modelos de espargidores aerossol e os respectivos órgãos alvos correspondentes:

Quadro 2. Comparativo das características dos modelos de aerossol.

\begin{tabular}{|c|c|c|c|c|}
\hline Efeito & Órgão alvo & Stream & Cone & Névoa \\
\hline $\begin{array}{c}\text { Irritação oculare } \\
\text { blefaroespasmo }\end{array}$ & Olhos & Sim & Sim & Não \\
\hline Lesão ocasionada pela pressão & Olhos & Sim & Sim & Não \\
\hline Irritação respiratória & Sistema respiratório & Não & Não* & Sim \\
\hline Broncoespasmo & Sistema respiratório & Não & Não* & Sim \\
\hline $\begin{array}{c}\text { Hemorragia pulmonar } \\
\text { ou enfisema alveolar }\end{array}$ & Sistema respiratório & Não & Não* & Sim \\
\hline Aspiração & Boca/garganta & Sim & Não & Não \\
\hline
\end{tabular}

Fonte: Adaptado ${ }^{4}$

* Alguns espargidores no modelo cone podem incluir partículas de tamanho capazes de entrar no sistema respiratório, neste caso eles poderão causar efeitos neste sistema.

\section{Conclusão}

A utilização de espargidor de agente químico lacrimogêneo em determinadas ocorrências onde o indivíduo abordado esboce uma resistência passiva é altamente recomendável. A prévia utilização do espargidor antes de se aplicar o nível de força denominado "controle de contato" irá favorecer, em muito, para a preservação da integridade física do(s) indivíduo(s) abordado(s), bem como do(s) policial(ais) que está(ão) empenhado(s) naquela ocorrência.

No entanto, para que o policial possa utilizar o espargidor, necessariamente precisa ser habilitado para manuseá-lo, ha- 
bilitação esta que geralmente é realizada durante os cursos de formação. Durante essa habilitação é de suma importância que o policial seja exposto ao agente químico lacrimogêneo pelos seguintes motivos: para que ele tenha conhecimento dos efeitos fisiológicos ocasionados pela exposição ao agente químico; para que possa ter controle emocional em uma eventual exposição ao agente químico durante o atendimento de uma ocorrência; e, para que possa descontaminar o indivíduo após ele ter sido imobilizado.

Contudo, como citado durante o presente artigo, não serão em todas as ocorrências em que for oferecida resistência passiva que o policial deverá utilizar o espargidor. $\mathrm{O}$ agente de segurança pública deverá analisar cada caso concreto com o intuito de verificar se estão presentes ou não motivos que justifiquem a utilização do espargidor, sob pena de responder administrativa e/ou penalmente pelo mau uso ou por algum excesso cometido.

Entendendo que seja o caso para utilização do espargidor, o policial deve ter conhecimento do alcance máximo do espargidor, uma vez que esta distância deve ser levada em conta para que o sujeito possa ser exposto a uma concentração eficaz do agente químico.

Importante ainda destacar que o espargidor que eventualmente será utilizado em uma ocorrência deve obedecer a todas as normas legais vigentes no Brasil, ou seja, ser fabricado por uma empresa idônea, registrada de acordo com as exigências legais e fiscalizada pelo Exército Brasileiro.

O policial ao utilizar o espargidor deve sempre ter em mente que este instrumento não é $100 \%$ eficaz. Em algumas ocorrências ele poderá não causar o efeito esperado no indivíduo exposto à solução lacrimogênea, assim, o policial deverá atuar com cautela, evitando ter uma falsa sensação de segurança.

Por fim, o método que o espargidor será acondicionado deve ser levado em conta, uma vez que se for exposto a altas temperaturas, como deixar no porta-luvas ou porta malas da viatura, pode ocasionar a explosão do recipiente do espargidor devido ao aumento da pressão interna do invólucro, bem como interferir na velocidade das partículas ao ser acionado.

Abaixo, é apresentada um quadro que resume os principais parâmetros de limites para os espargidores:
Quadro 3. Parâmetros de limites para os espargidores de agentes químicos lacrimogêneos.

\begin{tabular}{|c|c|c|c|}
\hline Efeito & Gravidade & Limite & Observações \\
\hline $\begin{array}{l}\text { Irritação ocular e } \\
\text { blefaroespasmo }\end{array}$ & 1 & $\begin{array}{c}50 \mu \mathrm{g} / \mathrm{L} \text { (blefaro- } \\
\text { espasmo) } \\
0,5 \mu \mathrm{g} / \mathrm{L} \text { (irritação } \\
\text { ocular de ter- } \\
\text { ceiros) }\end{array}$ & $\begin{array}{c}\text { Efeito previsto para es- } \\
\text { pargidores que disper- } \\
\text { sam grandes partículas } \\
\text { de aerossol }\end{array}$ \\
\hline $\begin{array}{l}\text { Irritação no trato res- } \\
\text { piratório }\end{array}$ & 1 & $\begin{array}{l}0,5 \mu \mathrm{g} \text { para a } \\
\text { região traque- } \\
\text { obronquial do } \\
\text { pulmão }\end{array}$ & $\begin{array}{l}\text { Efeito previsto para } \\
\text { espargidores que dis- } \\
\text { persam pequenas partí- } \\
\text { culas de aerossol }\end{array}$ \\
\hline $\begin{array}{l}\text { Broncoespasmo em } \\
\text { indivíduos normais }\end{array}$ & $1-3$ & $\begin{array}{l}>33 \mu g \text { para a } \\
\text { região traque- } \\
\text { obronquial do } \\
\text { pulmão }\end{array}$ & $\begin{array}{l}\text { Baseado em estudos } \\
\text { clínicos em humanos }\end{array}$ \\
\hline $\begin{array}{c}\text { Broncoespasmo em } \\
\text { asmáticos }\end{array}$ & $1-3$ & $\begin{array}{l}0,03 \mu \mathrm{g} \text { para a } \\
\text { região traque- } \\
\text { obronquial do } \\
\quad \text { pulmão }\end{array}$ & $\begin{array}{l}\text { Baseado em estudos } \\
\text { clínicos em humanos }\end{array}$ \\
\hline $\begin{array}{l}\text { Hemorragia pulmonar } \\
\text { ou enfisema alveolar }\end{array}$ & $2-3$ & $\begin{array}{c}8 \mu g \text { para a região } \\
\text { pulmonar }\end{array}$ & $\begin{array}{c}\text { Baseado em estudos } \\
\text { em animais }\end{array}$ \\
\hline $\begin{array}{l}\text { Lesão ocasionada } \\
\text { pela pressão das par- } \\
\text { tículas }\end{array}$ & $1-3$ & $\begin{array}{l}20 \text { psi - SE1 } \\
38 \text { psi - SE3 }\end{array}$ & Não \\
\hline Aspiração & $1-3$ & $\begin{array}{l}7 \mathrm{ml} \text { para um } \\
\text { adulto de } 70 \mathrm{~kg}\end{array}$ & $\begin{array}{l}\text { Baseado na aspiração } \\
\text { de fluídos por humanos }\end{array}$ \\
\hline Inflamabilidade & $1-3$ & $\begin{array}{l}\text { Dependente do } \\
\text { solvente e prope- } \\
\text { lente }\end{array}$ & $* * * * * * * * *$ \\
\hline
\end{tabular}

Fonte: Adaptado ${ }^{4}$

1 - lesão controlada que irá se curar sozinha;

2 - efeitos mais persistentes, sérios ou extensivos. Recomenda-se uma avaliação

médica/tratamento, mas ainda existe a possibilidade de cura sem a necessidade de uma intervenção especial;

3 - efeito potencialmente nocivo à saúde, gerando risco de morte ou risco de lesão permanente. Necessário hospitalização e/ou cuidados especiais.

\section{Referências}

1. Souza V. Policial é morto com a própria arma durante abordagem em SP. R7 [Internet]. 2018 Feb 25. Disponível em: https://noticias.r7.com/sao-paulo/policiale-morto-com-a-propria-arma-durante-abordagemem-sp-25022018. Acesso em: 12 de maio de 2019.

2. Secretaria Nacional de Segurança Pública - SENASP. Uso diferenciado da força. Brasília; 2016.

3. Sandes WF. O uso da força na formação de jovens tenentes: Um desafio para a atuação democrática da 
Polícia Militar de Mato Grosso [Dissertação]. Cuiabá: Universidade Federal de Mato Grosso; 2007. Mestrado em Educação.

4. Haber L, Nance P, Maier A, Price P, Olajos E, Bickford L, et al. HumaneEffectiveness and risk characterization of oleoresin capsicum (oc) and pelargonic acid vanillylamide (PAVA or Nonivamide) hand-held devices. Texas; 2008.

5. University of Guelph (UoG), et al. Pesticide safety for agricultural assistants student workbook. Ontario, Canada; 2004.

6. Adkins LD. Oleoresin capsicum: An analysus of the implementation of pepper spray into the law enforcement use of force continuum in a selected police department [Tese]. Tennessee: School of Graduate Studies, East Tennessee State University; 2003. Master of Arts in Criminal Justice and Criminology.

7. Brasil. Constituição da República Federativa do Brasil. Emenda Constitucional no 91, de 18 de fevereiro de 2016. Brasília, 5 out. 1988. Disponível em: http://www. planalto.gov.br/ccivil_03/constituicao/constituicao. htm. Acesso em: 16 de maio 2019.

8. Brasil. Decreto-Lei n 2.848 , de 7 de dezembro de 1940. Código Penal. Rio de Janeiro, 7 dez. 1940. Disponível em: http://www.planalto.gov.br/ccivil_03/decreto-lei/ del2848compilado.htm. Acesso em: 16 de maio 2019.

9. Brasil. Portaria Interministerial $n^{\circ} 4.226$, de 31 de dezembro de 2010. Estabelece Diretrizes sobre o Uso da Força pelos Agentes de Segurança Pública. Brasília, 31 dez. 2010. Disponível em: https://www.conjur.com. $\mathrm{br} / \mathrm{dl} /$ integra-portaria-ministerial.pdf. Acesso em: 16 de maio 2019.

10. Home Office. Comparison report on CS and PAVA Sprays. Publication Number: 24/14. Reino Unido; 2014.

11. Organização das Nações Unidas. Código de Conduta para Encarregados da Aplicação da Lei. Assembleia Geral, 1979. Disponível em: www.cpc.pm.rn.gov.br/ legislacao/legconduta.doc. Acesso em: 16 de maio de 2019.

12. Organização das Nações Unidas. Declaração Universal dos Direitos Humanos. Assembleia Geral, 1948. Disponível em: http://www.onu-brasil.org.br/documentos_direitoshumanos.php. Acesso em: 16 de maio de 2019.

13. Organização das Nações Unidas. Princípios Básicos sobre o Uso da Força e Armas de fogo. Havana, Cuba, 1990. Disponível em: www.cpc.pm.rn.gov.br/legislacao/legforca.doc. Acesso em: 16 de maio de 2019.
14. Phillips SW. TR-04-95 Oleoresin Capsicum in Buffalo. Canada: Canadian Police Research Centre; 1994.

15. Terrill W, Paoline EA. Examining Less Lethal Force Policy and the Force Continuum Results From a National Useof-Force Study. SAGE; 2012. 